\title{
circ-PRKCI targets miR-1294 and miR-186-5p by downregulating FOXK1 expression to suppress glycolysis in hepatocellular carcinoma
}

\author{
WENQI CHEN ${ }^{1,2}$, YUEHUA $\mathrm{LI}^{3}$, JING ZHONG $^{4}$ and GEBO WEN ${ }^{3,4}$
}

${ }^{1}$ Key Laboratory of Cancer Cellular and Molecular Pathology in Hunan Province, Cancer Research Institute, Hengyang Medical College, University of South China, Hengyang, Hunan 421001; ${ }^{2}$ Department of Clinical Oncology, The University of Hong Kong-Shenzhen Hospital, Shenzhen, Guangdong 518053; ${ }^{3}$ Key Laboratory of Cancer Cellular and Molecular Pathology in Hunan Province, Cancer Research Institute, Hengyang Medical College, University of South China, Hengyang, Hunan 421001; ${ }^{4}$ Institute of Clinical Medicine, First Affiliated Hospital of University of South China, Hengyang, Hunan 421001, P.R. China

Received October 13, 2020; Accepted January 29, 2021

DOI: $10.3892 / \mathrm{mmr} .2021 .12103$

\begin{abstract}
Numerous human circular RNAs (circRNAs/circ) have been functionally characterized. However, the potential role of circ-protein kinase $\mathrm{C}$ iota (PRKCI) in hepatocellular carcinoma (HCC) remains unknown. The effects of each transfection and expression levels of circ-PRKCI, microRNA (miR)-1294, miR-186-5p and forkhead box K1 (FOXK1) in HCC cells were analyzed using reverse transcription-quantitative PCR analysis. The interactions between circ-PRKCI and miR-1294 or miR-186-5p, and miR-1294 or miR-186-5p and FOXK1 were validated using dual luciferase reporter assays. The viability, invasion and migration of HCC cells were determined using Cell Counting Kit-8, Transwell and wound healing assays, respectively. The expression levels of FOXK1, hexokinase-2 (HK2), glucose transporter 1 (GLUT1) and lactate dehydrogenase A (LDHA) in HCC cells were analyzed using western blotting. The levels of glucose and lactic acid in the cultured supernatant were detected using commercially available kits. The results of the present study revealed that miR-1294 and miR-186-5p expression levels were downregulated in the HCC cell line, HCCLM3, and were subsequently downregulated by circ-PRKCI overexpression and upregulated by the knockdown of circ-PRKCI. circ-PRKCI overexpression promoted the viability, invasion and migration of HCCLM3 cells, which was also reversed by the overexpression of miR-1294 and miR-186-5p. In addition, the overexpression of circ-PRKCI upregulated FOXK1 expression levels, while the overexpression of miR-1294 and miR-186-5p downregulated
\end{abstract}

Correspondence to: Dr Gebo Wen, Institute of Clinical Medicine, First Affiliated Hospital of University of South China, 69 Chuanshan Road, Shigu, Hengyang, Hunan 421001, P.R. China

E-mail:wengb@usc.edu.cn

Key words: circular RNA-protein kinase C iota, microRNA-1294, microRNA-186-5p, forkhead box K1, hepatocellular carcinoma
FOXK1 expression levels. Conversely, the knockdown of circPRKCI expression downregulated FOXK1 expression levels, while the knockdown of miR-1294 and miR-186-5p upregulated FOXK1 expression levels. Furthermore, circ-PRKCI was identified to target miR-1294 and miR-186-5p, and miR-1294 and miR-186-5p were subsequently found to target FOXK1. The overexpression of circ-PRKCI also increased glucose and lactic acid levels, while the knockdown of FOXK1 decreased glucose and lactic acid levels. The knockdown of circ-PRKCI decreased glucose and lactic acid levels, which were reversed by FOXK1 overexpression. In conclusion, the findings of the present study suggested that circ-PRKCI may promote the viability, invasion and migration of HCC cells by sponging miR-1294 and miR-186-5p to upregulate FOXK1 expression levels.

\section{Introduction}

Primary liver cancer refers to the primary malignant cancer located in the hepatocytes or bile duct cells in the liver (1). In China, liver cancer has the fifth highest incidence rate (accounting for 9\% of all cancer-related incidences) and is the second leading cause of death (accounting for 13\% of all cancer-related mortalities); therefore, liver cancer has a significant impact on the life and health of individuals (2). Amongst the subtypes of liver cancer, hepatocellular carcinoma (HCC) has the highest incidence and accounts for $>80 \%$ of primary liver cancer types (3). Currently, there are various therapeutic approaches available for HCC, including surgical resection, cryotherapy, laser therapy and interventional surgery; however, due to the highly invasive and metastatic nature of $\mathrm{HCC}$, the median survival time of patients with advanced $\mathrm{HCC}$ is only 3-6 months, and the 5-year survival rate remains at $12.5 \%(4,5)$. Therefore, the pathological mechanisms of $\mathrm{HCC}$ at the molecular level should be further investigated to determine effective treatment targets for HCC.

Circular RNAs (circRNAs/circs) are a type of non-coding RNA formed by the covalent bonding between the 5 ' and 3 ' ends of linear precursor RNA through a reverse splicing 
mechanism, which is associated with gene transcription and post-transcriptional gene expression regulation (6). The expression levels of circ-protein kinase C iota (PRKCI) have been discovered to be upregulated in gastric cancer and promoted the proliferation, invasion and migration of gastric cancer cells (7). circ-PRKCI was also found to bind with microRNA (miRNA/miR) as a competitive RNA to promote the progression of esophageal squamous cell carcinoma (8). Furthermore, the upregulation of circ-PRKCI expression levels was discovered to enhance the proliferation and migration of HCC cells. The upregulated expression levels were also positively associated with an increase in infiltration depth and tumor node metastasis classification (9). In the present study, StarBase was used to predict the target genes of circ-PRKCI.

Notably, the upregulation of miR-1294 expression levels was demonstrated to inhibit the proliferation and promote the apoptosis of HCC cells (10). miR-186-5p expression levels were reported to be downregulated in HCC tissues and cells, which promoted the tumorigenesis and metastasis of HCC, while the overexpression of miR-186-5p suppressed the viability and increased the apoptosis and autophagy of the cancer cells $(11,12)$. In addition, multiple previous studies have suggested that both miR-1294 and miR-186-5p could regulate the expression levels of forkhead box K1 (FOXK1) $(13,14)$. FOXK1 expression levels were discovered to be upregulated in liver cancer cells, which regulated glycolysis and subsequently affected the viability of liver cancer cells (15).

However, whether circ-PRKCI can regulate FOXK1 through miR-1294 and miR-186-5p to affect glycolysis in HCC cells remains to be determined.

\section{Materials and methods}

Plasmids. For the stable overexpression (Ov) of circ-PRKCI and FOXK1, sequences were cloned into pcDNA3.1 vectors (Invitrogen; Thermo Fisher Scientific, Inc.); empty plasmids were used as the negative control (NC) for the overexpression plasmids. The following small interfering RNAs (siRNA/si) and miRNA oligonucleotides were obtained from Guangzhou RiboBio Co., Ltd.: Si-NC (cat. no. siB06525141922-1-5), si-circ-PRKCI-1 (cat. no. siB0804170942011-1-5), si-circPRKCI-2 (cat. no. siB0804170942012-1-5), si-FOXK1-1 (cat. no. siG151012011528-1-5), si-FOXK1-2 (cat. no. siG151012011536-1-5), mimic-NC (cat. no. miR1N0000001-1-5), miR-1294 mimic (cat. no. miR10005884-1-5), miR-186-5p mimic (cat. no. miR10000456-1-5), miR-1294 inhibitor (cat. no. miR20005884-1-5) and miR-186-5p inhibitor (cat. no. miR20000456-1-5).

Cell culture. The human hepatic epithelial cell line, THLE-3, was obtained from the American Type Culture Collection and the HCC cell line, HCCLM3, was purchased from Procell Life Science \& Technology Co., Ltd. THLE-3 and HCCLM3 cells were cultured in DMEM (Gibco; Thermo Fisher Scientific, Inc.) supplemented with 10\% FBS (Gibco; Thermo Fisher Scientific, Inc.), $100 \mathrm{U} / \mathrm{ml}$ penicillin and $100 \mu \mathrm{g} / \mathrm{ml}$ streptomycin, and maintained in a humidified atmosphere at $37^{\circ} \mathrm{C}$ with $5 \% \mathrm{CO}_{2}$.

Cell transfection. HCCLM3 cells were cultured until reaching the logarithmic stage and then seeded at a cell density of
$1 \times 10^{5}$ cells/ml into a 6 -well plate. The cells were subsequently incubated in an incubator at $37^{\circ} \mathrm{C}$ for $24 \mathrm{~h}$ until the cells adhered to the wall and the confluence reached $70 \%$. The HCCLM3 cells were subsequently transfected with plasmids, siRNAs or oligonucleotides using Lipofectamine ${ }^{\circledR} 2000$ transfection reagent (Invitrogen; Thermo Fisher Scientific, Inc.) at $37^{\circ} \mathrm{C}$ for $48 \mathrm{~h}$, and cells then were used for subsequent experimentation.

Reverse transcription-quantitative PCR (RT-qPCR). Total RNA was extracted from cells using TRIzol ${ }^{\circledR}$ reagent (Invitrogen; Thermo Fisher Scientific, Inc.) A total of $2 \mu \mathrm{g}$ RNA was reverse transcribed into cDNA using a Transcriptor First Strand cDNA Synthesis kit (Thermo Fisher Scientific, Inc.) according to the manufacturer's protocol. qPCR was subsequently performed using a SYBR-Green Real-time PCR kit (Invitrogen; Thermo Fisher Scientific, Inc.), according to the manufacturer's protocol. The following thermocycling conditions were used for the qPCR: Initial denaturation at $95^{\circ} \mathrm{C}$ for $5 \mathrm{~min}$; followed by 40 cycles of denaturation at $95^{\circ} \mathrm{C}$ for $15 \mathrm{sec}$, annellation at $60^{\circ} \mathrm{C}$ for $15 \mathrm{sec}$ and extension at $72^{\circ} \mathrm{C}$ for $45 \mathrm{sec}$. The following primer sequences were used for the qPCR: circPRKCI forward, 5'-CGGAGGTTCCAGCTCGTTAGTC-3' and reverse, 5'-GCAACCGGAATGTGGAATTGA-3'; FOXK1 forward, 5'-GCCACAAAGGCTGGCAGAATT-3' and reverse, 5'-TGGCTTCAGAGGCAGGGTCTAT-3'; GAPDH forward, 5'-TCGACAGTCAGCCGCATCTT-3' and reverse, 5'-GAGTTAAAAGCAGCCCTGGTG-3'; miR-1294 forward, 5'-TATGATCTCACCGAGTCCT-3' and reverse, 5'-CACCTTCCTAATCCTCAGTT-3'; miR-186-5p forward, 5'-AAGAATTCTCCTTTTGGGCT-3' and reverse, 5'-GTGCGTGTCGTGGAGTCG-3'; and U6 forward, 5'-AGGTGTGTGCTGCTATGAAC-3' and reverse, 5'-CAGGGCATTTGTCATTATTG-3'. GAPDH or U6 were used as the internal reference genes for mRNA and miRNA, respectively, and the $2^{-\Delta \Delta C q}$ method (16) was used to quantify the relative expression levels of circ-PRKCI, miR-1294, miR186-5p and FOXK1.

Dual luciferase reporter assay. circ-PRKCI-wild-type (WT), circ-PRKCI-mutant (MUT), FOXK1-WT and FOXK1-MUT 3'-untranslated region (UTR) sequences were cloned into pmirGLO plasmids (Promega Corporation). The pmirGLO plasmids were subsequently co-transfected into HCCLM3 cells with the miR-1294, miR-186-5p mimic or miR-NC using Lipofectamine 2000 reagent. After transfection for $48 \mathrm{~h}$, the relative luciferase (Luc) activity was measured using a Dual Luciferase Reporter assay system (Promega Corporation) and the Luc value was normalized to the Renilla (R)-Luc value.

Cell Counting Kit-8 (CCK-8) assay. HCCLM3 cells from each group were plated into a 96 -well plate $\left(5 \times 10^{3}\right.$ cells/well) and cultured overnight at $37^{\circ} \mathrm{C}$. Following incubation for $24 \mathrm{~h}$, the medium was removed and HCCLM3 cells were rinsed with PBS. Subsequently, $10 \mu$ l CCK-8 solution (Beyotime Institute of Biotechnology) was added/well and incubated at $37^{\circ} \mathrm{C}$ with $5 \% \mathrm{CO}_{2}$ for $2 \mathrm{~h}$. The absorbance of each group at $24 \mathrm{~h}$ was measured at a wavelength of $450 \mathrm{~nm}$ with a microplate reader to reflect the change in the cell viability. 
Wound healing assay. HCCLM3 cells from each group were seeded into 12 -well plates $\left(5 \times 10^{4}\right.$ cells/well) and cultured in DMEM with $5 \% \mathrm{FBS}$ at $37^{\circ} \mathrm{C}$. After reaching $100 \%$ confluence, a 200- $\mu 1$ sterile pipette tip was used to scratch the confluent monolayer; the cells were then washed with PBS to remove the non-adherent cells. The cells were subsequently cultured with serum-free DMEM at $37^{\circ} \mathrm{C}$ for $24 \mathrm{~h}$. The wound area at 0 and $24 \mathrm{~h}$ was imaged using a light microscope (magnification, x100).

Transwell assay. A total of $3 \times 10^{4}$ cells from each group were seeded in serum-free DMEM into the upper chamber of Transwell plates precoated with Matrigel at $37^{\circ} \mathrm{C}$ for $30 \mathrm{~min}$ and then cultured at $37^{\circ} \mathrm{C}$ for $24 \mathrm{~h}$. DMEM supplemented with $10 \%$ FBS was plated into the lower chamber. Following incubation for $24 \mathrm{~h}$ at $37^{\circ} \mathrm{C}$, the cells remaining on the upper surface of the membrane were removed. The invasive cells in the lower chamber were fixed with $4 \%$ paraformaldehyde at room temperature for $30 \mathrm{~min}$ and stained with $0.2 \%$ crystal violet solution for $10 \mathrm{~min}$ at room temperature. Finally, the membrane was washed with PBS once and visualized under a light microscope (magnification, x100).

Western blotting. HCCLM3 cells from each group were collected and total protein was extracted using RIPA lysis buffer (Beyotime Institute of Biotechnology) at 3,000 x g at $4^{\circ} \mathrm{C}$ for $15 \mathrm{~min}$. Total protein was quantified using a BCA assay kit (Pierce; Thermo Fisher Scientific, Inc.) and $20 \mu \mathrm{g}$ protein/lane was separated via $12 \%$ SDS-PAGE. The separated proteins were transferred onto PVDF membranes and blocked at room temperature with 5\% skimmed milk for $30 \mathrm{~min}$. The membranes were then incubated overnight at $4^{\circ} \mathrm{C}$ with the following primary antibodies: Anti-FOXK1 (1:1,000; cat. no. ab18196; Abcam), anti-hexokinase-2 (HK2; 1:1,000; cat. no. ab209847; Abcam), anti-glucose transporter 1 (GLUT1; 1:200; cat. no. ab150299; Abcam), anti-lactate dehydrogenase A (LDHA; 1:1,000; cat. no. ab101562; Abcam) and anti-GAPDH (1:1,000; cat. no. ab8245; Abcam). Following the primary antibody incubation, the membranes were incubated with an anti-rabbit IgG HRP-conjugated antibody $(1: 2,000$; cat. no. ab6721; Abcam) or anti-mouse IgG HRP-conjugated antibody (1:1,000; cat. no. 7076; Cell Signaling Technology, Inc.) for $1 \mathrm{~h}$ at room temperature. Protein bands were visualized in a darkroom using ECL reagent (Bio-Rad Laboratories, Inc.). Densitometric analysis was performed using Image-Pro Plus software (version 6.0; Media Cybernetics, Inc.)

Detection of glucose and lactic acid levels. Following transfection for $24 \mathrm{~h}$, HCCLM3 cells were centrifuged at 3,000 x g at $4^{\circ} \mathrm{C}$ for $15 \mathrm{~min}$ to obtain the supernatant. The levels of glucose and lactic acid in the supernatant of each group were analyzed with a Glucose Assay kit (cat. no. E-BC-K234-S; Elabscience) and a L-Lactic Acid Colorimetric Assay kit (cat. no. E-BC-K044-S; Elabscience), according to the manufacturers' protocols, respectively, as previously described $(17,18)$.

Statistical analysis. All experiments were repeated three times. Statistical analysis was performed using SPSS 18.0 software (SPSS, Inc.) and data are presented as the mean \pm SD. Statistical differences between two groups were determined using an unpaired Student's t-test, while a one-way ANOVA with a Tukey's post hoc test was used to determine the statistical differences between multiple groups. $\mathrm{P}<0.05$ was considered to indicate a statistically significant difference.

\section{Results}

circ-PRKCI regulates the expression levels of miR-1294 and miR-186-5p in HCC cells. circ-PRKCI expression levels were significantly upregulated in HCCLM3 cells transfected with Ov-circ-PRKCI vector compared with the control and Ov-NC groups (Fig. 1A), while circ-PRKCI expression levels were downregulated in HCCLM3 cells transfected with si-circPRKCI-1 and si-circ-PRKCI-2 compared with the control and si-NC groups (Fig. 1B). circ-PRKCI expression levels were lower in HCCLM3 cells transfected with si-circ-PRKCI-1, thus si-circ-PRKC1-1 was selected for use in subsequent experiments. The expression levels of miR-1294 (Fig. 1C) and miR-186-5p (Fig. 1D) in HCCLM3 cells were downregulated compared with THLE-3 cells. circ-PRKCI overexpression significantly downregulated the expression levels of miR-1294 compared with the Ov-NC and control groups (Fig. 1E) and miR-186-5p (Fig. 1F), while the knockdown of circ-PRKCI upregulated the expression levels of miR-1294 (Fig. 1G) and miR-186-5p (Fig. 1H) in HCCLM3 cells compared with the control and si-NC groups.

circ-PRKCI targets $m i R-1294$ and $m i R-186-5 p$. The predicted binding sites between circ-PRKCI and miR-1294 are shown in Fig. 2A. The relative Luc activity of cells was significantly decreased following the co-transfection of cells with miR-1294 mimic and circ-PRKCI-WT compared with cells co-transfected with miR-NC and circ-PRKCI-WT. There were no significant differences observed in the relative luciferase activity of MUT reporter plasmids between cells transfected with the miRNA mimics and miR-NCs (Fig. 2B). The predicted binding sites between circ-PRKCI and miR-186-5p are presented in Fig. 2C. The relative Luc activity was significantly decreased following the co-transfection of cells with miR-186-5p mimic and circ-PRKCI-WT compared with cells co-transfected with miR-NC and circ-PRKCI-WT (Fig. 2D).

circ-PRKCI promotes the viability, invasion and migration of HCC cells by targeting miR-1294 and miR-186-5p. Following the transfection of HCCLM3 cells with the miR-1294 mimic or miR-186-5p mimic, the expression levels of miR-1294 (Fig. 3A) and miR-186-5p (Fig. 3B), respectively, were upregulated in HCCLM3 cells compared with cells transfected with the mimic-NC or the control group. Following the transfection of HCCLM3 cells with the miR-1294 inhibitor or miR-186-5p inhibitor, the expression levels of miR-1294 (Fig. 3C) and miR186-5p (Fig. 3D), respectively, were downregulated in HCCLM3 cells compared with cells transfected with inhibitor-NC or the control group. circ-PRKCI overexpression significantly promoted the viability of HCCLM3 cells compared with the Ov-NC and control groups, which was subsequently reversed following the overexpression of miR-1294 or miR-186-5p (Fig. 3E). The inhibition of circ-PRKCI also significantly suppressed the viability of HCCLM3 cells compared with the si-NC and control groups, which was partially reversed 
A

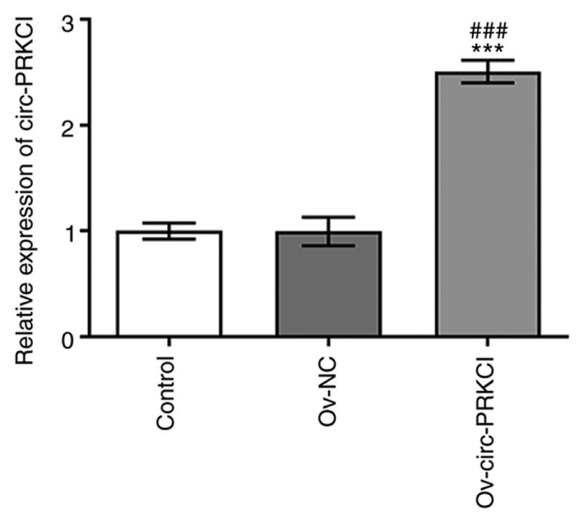

C

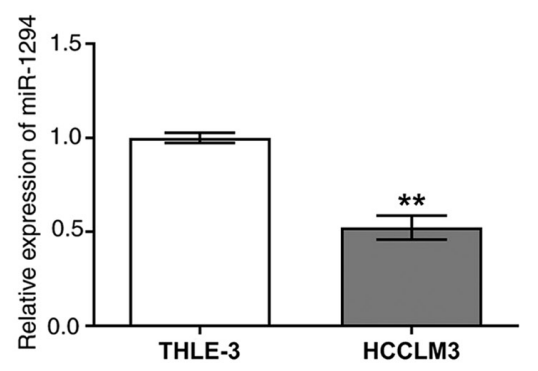

E

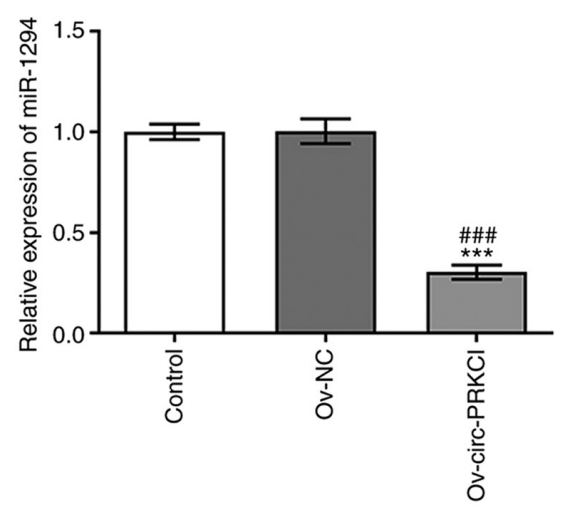

G

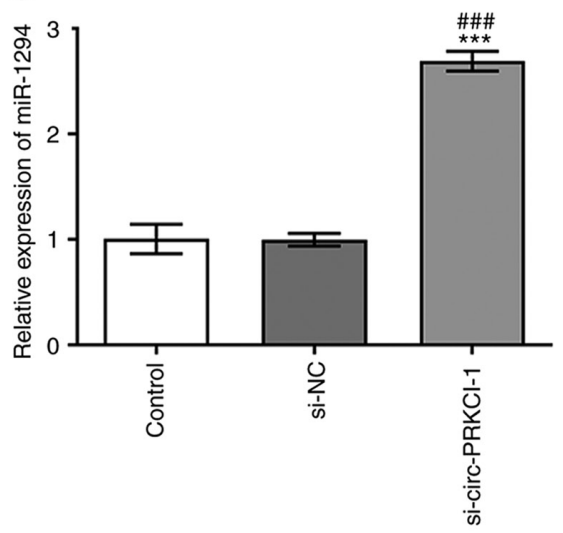

B

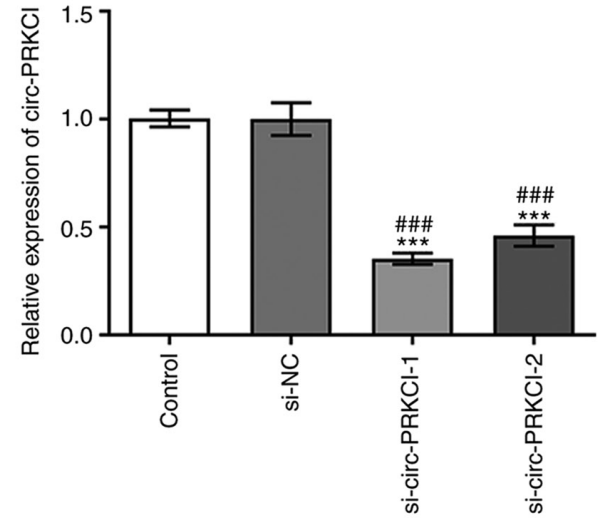

D

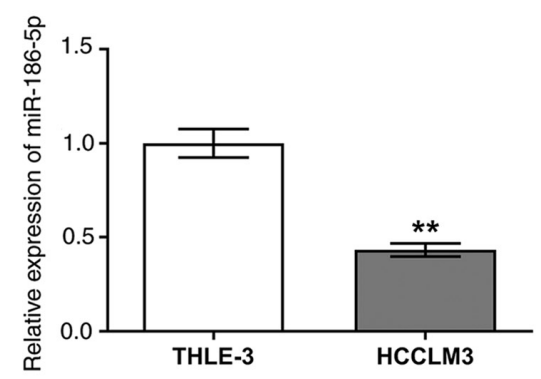

$\mathrm{F}$

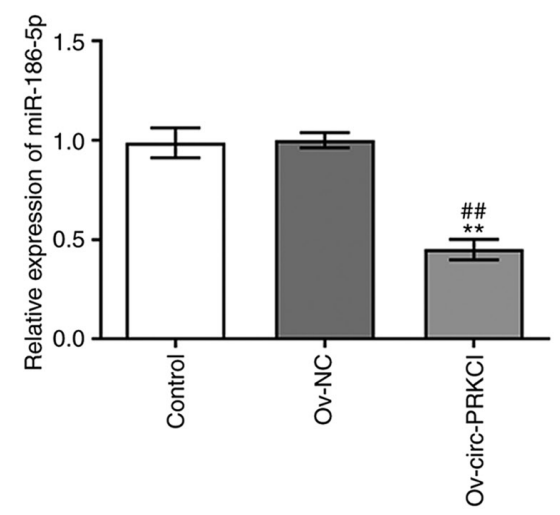

$\mathrm{H}$

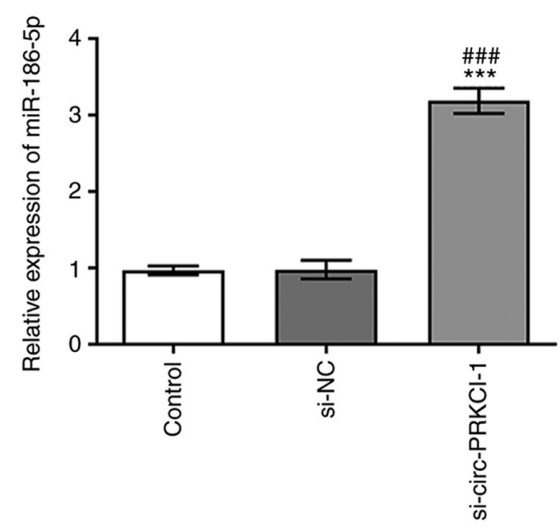

Figure 1. circ-PRKCI regulates the expression levels of miR-1294 and miR-186-5p in HCC cells. (A) circ-PRKCI expression levels in HCCLM3 cells following the transfection with Ov-circ-PRKCI were analyzed using RT-qPCR. ${ }^{* * *} \mathrm{P}<0.001$ vs. Control; ${ }^{\# \# \#} \mathrm{P}<0.001$ vs. Ov-NC. (B) circ-PRKCI expression levels in HCCLM3 cells following the transfection with si-circ-PRKCI-1 or si-circ-PRKCI-2 were analyzed using RT-qPCR. ${ }^{* * *} \mathrm{P}<0.001 \mathrm{vs}$. Control; ${ }^{\# \# *} \mathrm{P}<0.001$ vs. si-NC. Expression levels of (C) miR-1294 and (D) miR-186-5p in HCCLM3 and THLE-3 cells were analyzed using RT-qPCR. ${ }^{* *} \mathrm{P}<0.01$ vs. THLE-3. Expression levels of (E) miR-1294 and (F) miR-186-5p in HCCLM3 cells following the transfection with Ov-circ-PRKCI were analyzed using RT-qPCR. ${ }^{* *} \mathrm{P}<0.01,{ }^{* * *} \mathrm{P}<0.001$ vs. Control; ${ }^{\# \#} \mathrm{P}<0.01,{ }^{\# \# \#} \mathrm{P}<0.001$ vs. Ov-NC. Expression levels of $(\mathrm{G})$ miR-1294 and $(\mathrm{H}) \mathrm{miR}-186-5 \mathrm{p}$ in HCCLM3 cells following transfection with si-circ-PRKCI-1 were analyzed using RT-qPCR. ${ }^{* * *} \mathrm{P}<0.001$ vs. Control; ${ }^{\# \# "} \mathrm{P}<0.001$ vs. si-NC. circ, circular RNA; PRKC1, protein kinase C iota; RT-qPCR, reverse transcription-quantitative PCR; si, small interfering RNA; NC, negative control; miR, microRNA; Ov, overexpression. 

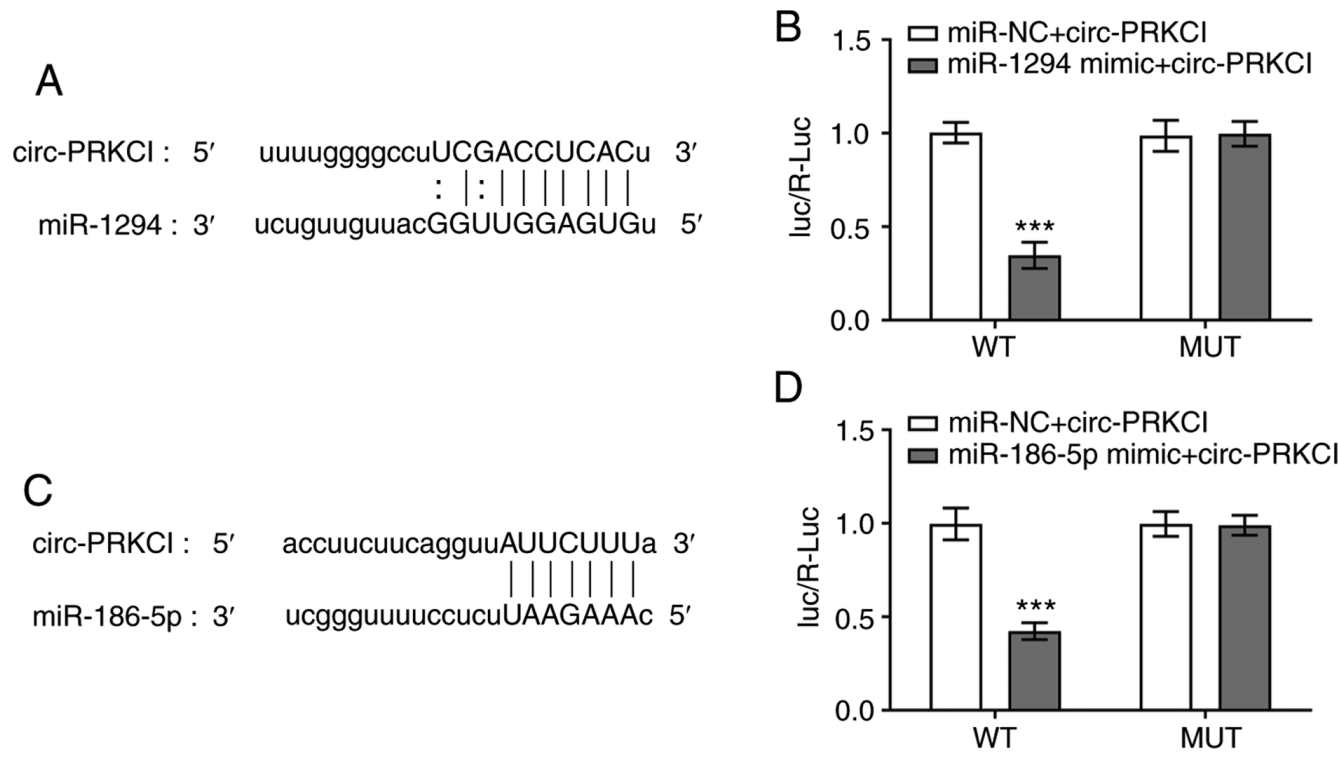

Figure 2. circ-PRKCI targets miR-1294 and miR-186-5p. (A) Binding sites between circ-PRKCI and miR-1294 are shown. (B) Dual luciferase reporter assay was used to validate the direct binding relationship between circ-PRKCI and miR-1294. (C) Binding sites between circ-PRKCI and miR-186-5p are shown. (D) Dual luciferase reporter assay was used to validate the direct binding relationship between circ-PRKCI and miR-186-5p. ${ }^{* * *} \mathrm{P}<0.001$ vs. miR-NC + circPRKCI WT. circ, circular RNA; PRKC1, protein kinase C iota; miR, microRNA; NC, negative control; WT, wild-type; MUT, mutant.

by miR-1294 inhibition or miR-186-5p inhibition (Fig. 3F). As shown in Fig. 3G-J, circ-PRKCI overexpression significantly promoted the migration and invasion of HCCLM3 cells compared with the $\mathrm{Ov}-\mathrm{NC}$ and control groups, which was subsequently reversed following the overexpression of miR-1294 or miR-186-5p. Conversely, the inhibition of circPRKCI significantly suppressed the migration and invasion of HCCLM3 cells compared with the Ov-NC and control groups, which was subsequently reversed following the knockdown of miR-1294 or miR-186-5p (Fig. 3K-N)

circ-PRKCI regulates FOXK1 expression levels by targeting miR-1294 and miR-186-5p. The overexpression of circ-PRKCI upregulated the expression levels of FOXK1 compared with the control and Ov-NC groups, while the promoting effect of circ-PRKCI overexpression on FOXK1 expression levels was significantly suppressed by the overexpression of miR-1294 or miR-186-5p (Fig. 4A). The knockdown of circ-PRKCI expression significantly downregulated FOXK1 expression levels compared with the si-NC and control groups, which was partially reversed following the knockdown by miR-1294 or miR-186-5p (Fig. 4B). The predicted binding sites between FOXK1 and miR-1294 are shown in Fig. 4C. The Luc/R-Luc value was significantly decreased in HCCLM3 cells co-transfected with the miR-1294 mimic and FOXK1-WT vector compared with the cells co-transfected with miR-NC and FOXK1-WT. There were no significant differences observed in the relative luciferase activity of MUT reporter plasmids between cells transfected with the miRNA mimics and miR-NCs (Fig. 4D). The binding sites between FOXK1 and miR-186-5p are presented in Fig. 4E. The Luc/R-Luc value was also significantly decreased in HCCLM3 cells co-transfected with the miR-186-5p mimic and FOXK1-WT compared with the cells co-transfected with miR-NC and FOXK1-WT (Fig. 4F).
circ-PRKCI suppresses glycolysis in HCC cells by regulating FOXK1 expression. FOXK1 expression levels were significantly upregulated in HCCLM3 cells transfected with Ov-FOXK1 compared with the Ov-NC and control groups (Fig. 5A). The mRNA expression levels of FOXK1 were also significantly downregulated in HCCLM3 cells transfected with si-FOXK1-1 and si-FOXK1-2 compared with the si-NC and control groups (Fig. 5B). The overexpression of circPRKCI significantly increased the levels of glucose (Fig. 5C) and lactic acid (Fig. 5E) compared with Ov-NC and control groups, which were subsequently reversed following the knockdown of FOXK1. Conversely, the knockdown of circPRKCI expression levels significantly decreased the levels of glucose (Fig. 5D) and lactic acid (Fig. 5F) compared with the $\mathrm{Ov}-\mathrm{NC}$ and control groups, which was subsequently reversed following the overexpression of FOXK1. As shown in Fig. 5G, circ-PRKCI overexpression upregulated the expression levels of HK2, GLUT1 and LDHA compared with the Ov-NC and control groups, which were subsequently reversed by FOXK1 inhibition. Conversely, the knockdown of circ-PRKCI expression significantly downregulated the expression levels of HK2, GLUT1 and LDHA compared with the si-NC and control groups, which were subsequently reversed by the overexpression of FOXK1 (Fig. 5H).

\section{Discussion}

It is estimated that $\sim 50 \%$ of ATP in tumor cells is synthesized through glycolysis (19). Although aerobic glycolysis occurs at low levels, it is a rapid process. The intermediate products can provide nutrients for cell proliferation and protect tumor cells, thereby promoting the excessive proliferation and metastasis of malignant tumor cells $(20,21)$. Therefore, the effective inhibition of glycolysis may suppress the progression of liver cancer. 
A
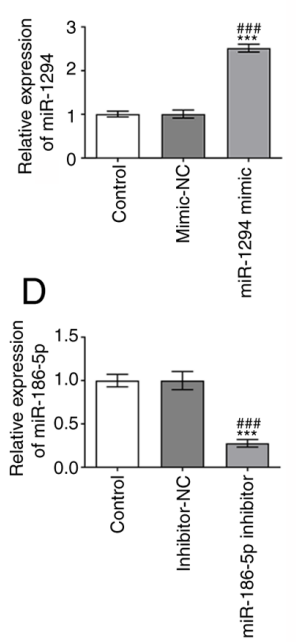

G
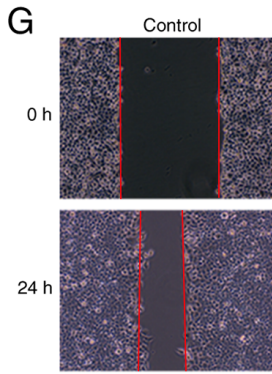

I

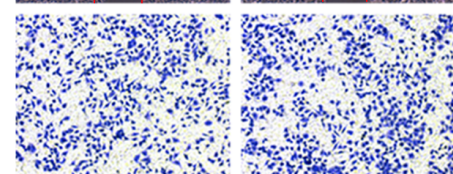

s.

$\mathrm{K}$

Control
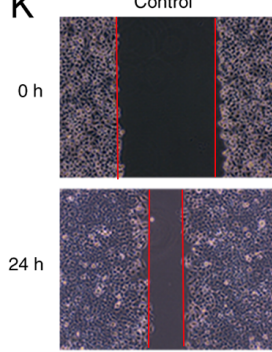

$\mathrm{M}$

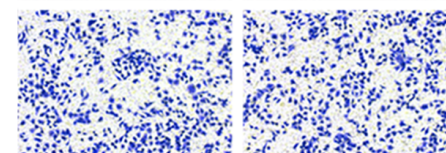

Ov-NC
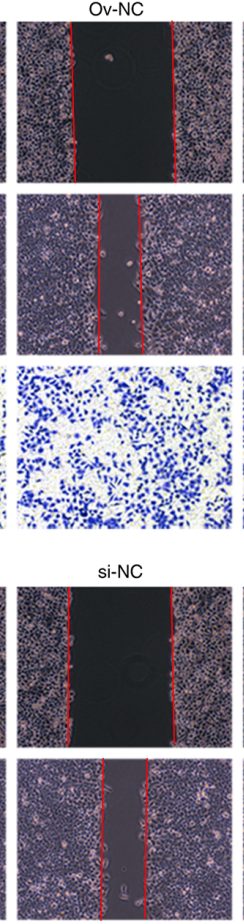

B

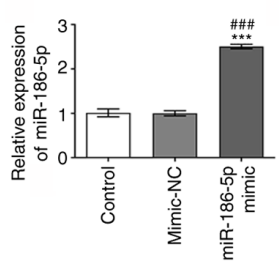

E

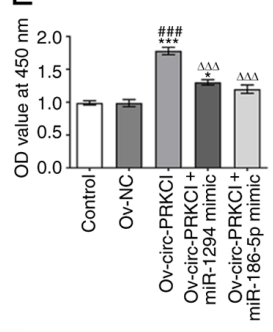

C

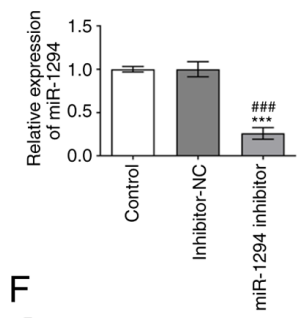

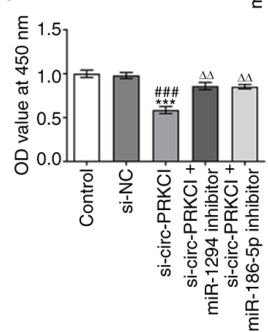
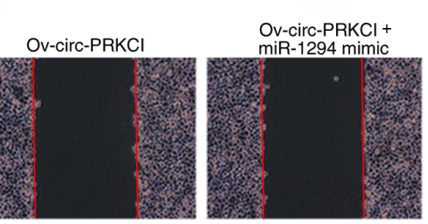

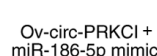
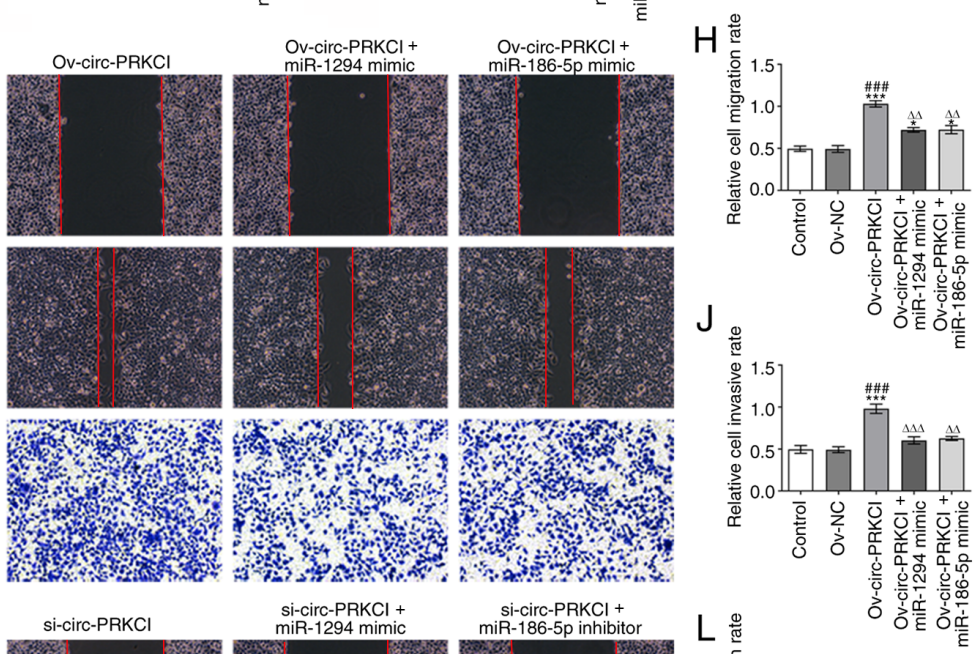

si-circ-PRKCl
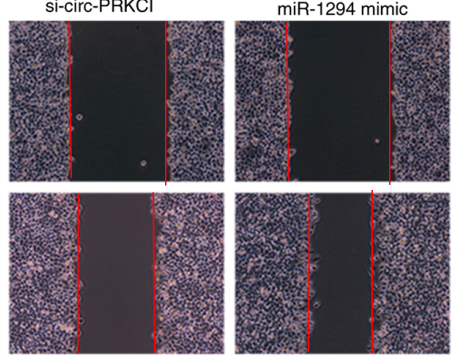

si-circ-PRKCl+
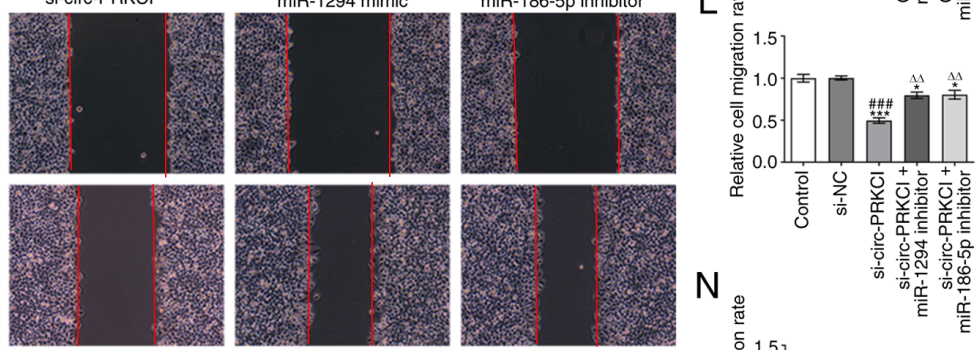

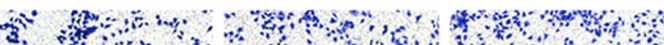

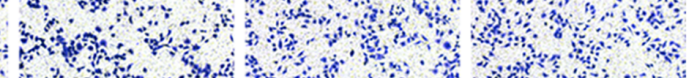

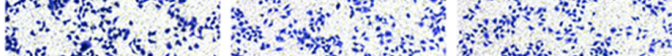
Non

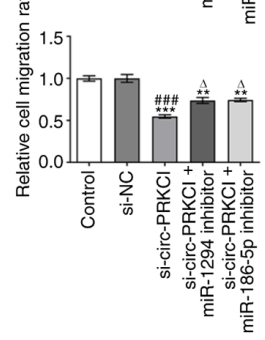

Figure 3. circ-PRKCI promotes the viability, invasion and migration of hepatocellular carcinoma cells by targeting miR-1294 and miR-186-5p. Expression levels of (A) miR-1294 and (B) miR-186-5p in HCCLM3 cells following the transfection with miR-1294 mimic or miR-186-5p mimic, respectively, were analyzed using RT-qPCR. ${ }^{* * *} \mathrm{P}<0.001$ vs. Control; ${ }^{\# \# \#} \mathrm{P}<0.001$ vs. mimic-NC. Expression levels of (C) miR-1294 and (D) miR-186-5p in HCCLM3 cells following the transfection with miR-1294 inhibitor or miR-186-5p inhibitor were analyzed using RT-qPCR. ${ }^{* * *} \mathrm{P}<0.001$ vs. Control; ${ }^{\# \# \#} \mathrm{P}<0.001$ vs. inhibitor-NC. (E) Viability of HCCLM3 cells following the transfection with Ov-circ-PRKCI and/or miR-1294 mimic or miR-186-5p mimic was analyzed using a CCK-8 assay. ${ }^{*} \mathrm{P}<0.05,{ }^{* * *} \mathrm{P}<0.001$ vs. Control; ${ }^{\# \# \#} \mathrm{P}<0.001$ vs. Ov-NC; ${ }^{\Delta \Delta} \mathrm{P}<0.001$ vs. Ov-circ-PRKCI. (F) Viability of HCCLM3 cells following the transfection with si-circ-PRKCI and/or miR-186-5p inhibitor or miR-186-5p inhibitor was analyzed using a CCK-8 assay. ${ }^{* * *} \mathrm{P}<0.001$ vs. Control; ${ }^{\# \# \#} \mathrm{P}<0.001$ vs. si-NC; ${ }^{\Delta \Delta} \mathrm{P}<0.01$ vs. si-circ-PRKCI. (G and H) Migration of HCCLM3 cells following the transfection with Ov-circ-PRKCI and/or miR-1294 mimic or miR-186-5p mimic was determined using a wound healing assay (magnification, $\mathrm{x} 100$ ). ${ }^{*} \mathrm{P}<0.05,{ }^{* * *} \mathrm{P}<0.001$ vs. Control; ${ }^{\# \# \#} \mathrm{P}<0.001$ vs. Ov-NC; ${ }^{\Delta \Delta} \mathrm{P}<0.01$ vs. Ov-circ-PRKCI. (I and J) Invasion of HCCLM3 cells following the transfection with Ov-circ-PRKCI and miR-1294 mimic or miR-186-5p mimic was determined using a Transwell assay (magnification, $\mathrm{x} 100$ ). ${ }^{* * *} \mathrm{P}<0.001$ vs. Control; ${ }^{\# \# \#} \mathrm{P}<0.001$ vs. Ov-NC; ${ }^{\Delta \Delta} \mathrm{P}<0.01,{ }^{\Delta \Delta \Delta} \mathrm{P}<0.001$ vs. Ov-circ-PRKCI. (K and L) Migration of HCCLM3 cells following the transfection with si-circ-PRKCI and miR-1294 inhibitor or miR-186-5p inhibitor was determined using a wound healing assay (magnification, $\mathrm{x} 100$ ). ${ }^{*} \mathrm{P}<0.05,{ }^{* * * *} \mathrm{P}<0.001$ vs. Control; ${ }^{\# \# \#} \mathrm{P}<0.001$ vs. si-NC; ${ }^{\Delta} \mathrm{P}<0.01$ vs. si-circ-PRKCI. (M and $\mathrm{N}$ ) Invasion of HCCLM3 cells following the transfection with si-circ-PRKCI and miR-1294 inhibitor or miR-186-5p inhibitor was determined using a Transwell assay (magnification, $\mathrm{x} 100)$. ${ }^{* *} \mathrm{P}<0.01$, ${ }^{* * *} \mathrm{P}<0.001$ vs. Control; ${ }^{\# \#} \mathrm{P}<0.001$ vs. si-NC; ${ }^{\stackrel{P}{P}<0.05}$ vs. si-circ-PRKCI. circ, circular RNA; PRKC1, protein kinase C iota; miR, microRNA; NC, negative control; RT-qPCR, reverse transcription-quantitative PCR; CCK-8, Cell Counting Kit-8; si, small interfering RNA; Ov, overexpression; OD, optical density. 
A
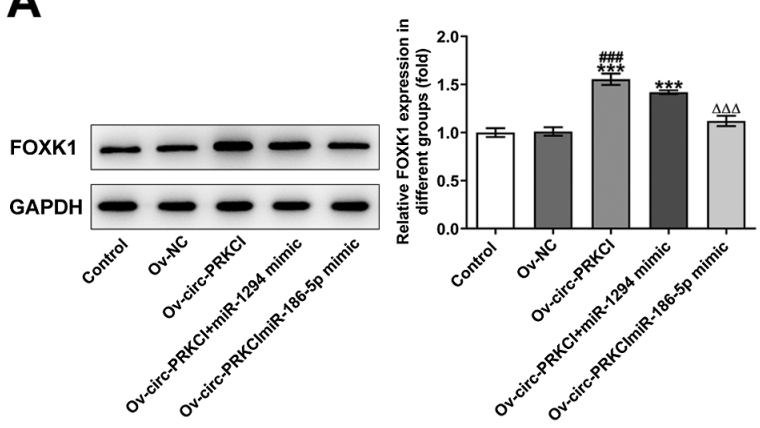

B

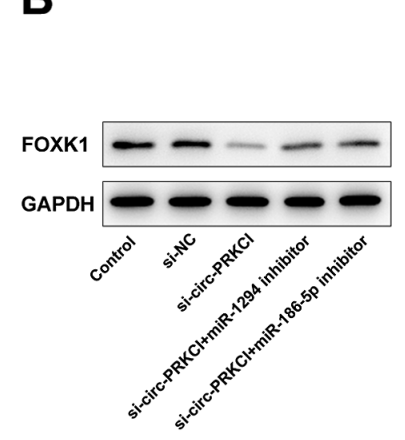

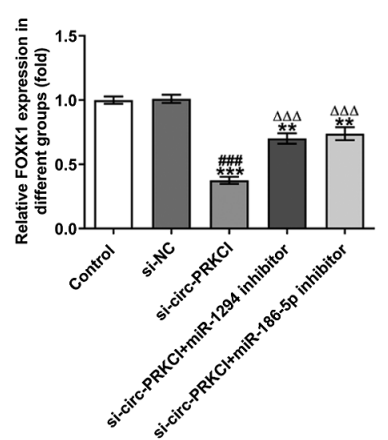

C $\begin{array}{ccccc}\text { FOXK1 } & : & 5 & \text { guuuugcuucagaAGCCUCACa } \\ \text { I:IIIIIII } & 3^{\prime} \\ \text { miR-1294 } & : & 3, & \text { ucuguuguuacggUUGGAGUGu } & \text { 5' }\end{array}$

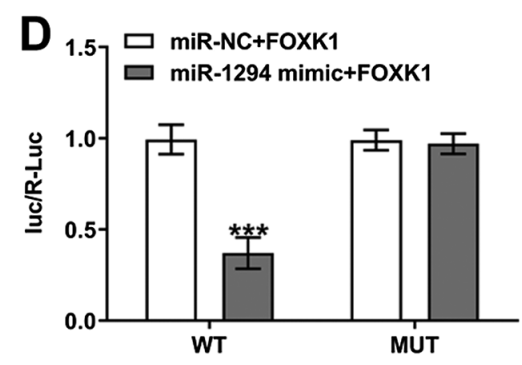

E

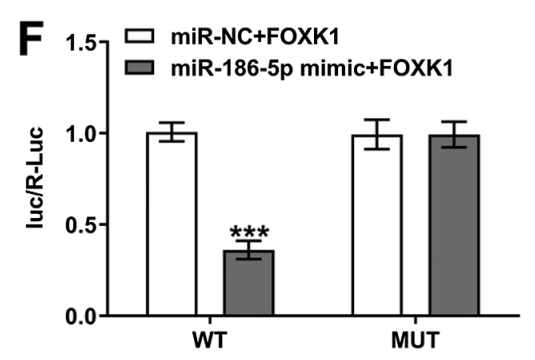

Figure 4. circ-PRKCI regulates FOXK1 expression levels by targeting miR-1294 and miR-186-5p. (A) FOXK1 expression levels in HCCLM3 cells following the transfection with Ov-circ-PRKCI and miR-1294 mimic or miR-186-5p mimic were analyzed using western blotting. ${ }^{* * *} \mathrm{P}<0.001$ vs. Control; ${ }^{\# \# \# ~} \mathrm{P}<0.001$ vs. Ov-NC; ${ }^{\Delta \Delta \Delta} \mathrm{P}<0.001$ vs. Ov-circ-PRKCI. (B) FOXK1 expression levels in HCCLM3 cells following the transfection with si-circ-PRKCI and miR-1294 inhibitor or miR-186-5p inhibitor were analyzed using western blotting. ${ }^{* *} \mathrm{P}<0.01,{ }^{* * *} \mathrm{P}<0.001$ vs. Control; ${ }^{\# \# \#} \mathrm{P}<0.001$ vs. si-NC; ${ }^{\Delta \Delta \Delta} \mathrm{P}<0.001$ vs. si-circ-PRKCI. (C) Binding sites between FOXK1 and miR-1294 are shown. (D) Dual luciferase reporter assay was used to validate the direct binding relationship between FOXK1 and miR-1294. ${ }^{* * *} \mathrm{P}<0.001$ vs. miR-NC + FOXK1 WT. (E) Binding sites between FOXK1 and miR-186-5p are shown. (F) Dual luciferase reporter assay was used to validate the direct binding relationship between FOXK1 and miR-186-5p. ${ }^{* * *} \mathrm{P}<0.001$ vs. miR-NC + FOXK1 WT. circ, circular RNA; PRKCI, protein kinase C iota; FOXK1, forkhead box K1; miR, microRNA; Ov, overexpression; NC, negative control; WT, wild-type; MUT, mutant; R, Renilla; Luc, luciferase.

Cancer is increasingly being regarded as a metabolismrelated disease, and tumor-related energy regulation has been investigated as a biochemical pathway and drug target for tumor therapy (22). In the past decade, molecular and cellular studies have highlighted the association between oncogenes, tumor suppressors and cancer glycolysis (23). For example, Guo et al (24) reported that miR-199a-5p directly targeted HK2 to suppress glycolysis and the tumorigenesis of liver cancer cells. The expression levels of pyruvate kinase M1/2 (PKM2) were also found to be highly expressed in HCC, which was essential for the aerobic glycolysis of HCC cells (25). Liang et al (26) demonstrated that the expression levels of miR-122 were closely associated with numerous glycolytic genes, such as PKM2, hexokinase, LDHA and GLUT1, through the comprehensive gene expression analysis of 94 liver cancer tissues. The same study also revealed that miR-122 targeted PKM2, which affected the metabolism of HCC. Numerous previous studies have reported the role of miR-186 in mediating glycolysis in several types of cancer; for example, miR-186 inhibited aerobic glycolysis in osteosarcoma cells by decreasing hypoxia-inducible factor 1 expression levels (27); circ-nuclear receptor interacting protein 1 promoted glycolysis and gastric cancer progression by downregulating miR-186-5p expression levels (28); and FOXK1 and/or FOXK2 were discovered to regulate aerobic glycolysis (29). In the present study, bioinformatics analysis predicted that circ-PRKCI targeted miR-186-5p and in turn, miR-186-5p targeted FOXK1. circ-PRKCI was revealed to suppress glycolysis in HCC cells by upregulating FOXK1 expression levels via miR-186-5p.

Previous studies reported that miR-1294 expression levels were downregulated in gastric, esophageal, epithelial ovarian and cervical cancers, while miR-1294 overexpression inhibited the cell proliferation and cell cycle progression of cancer cells (13,30-32). The results of the current study revealed that the expression levels of miR-1294 and miR-186-5p were downregulated in HCC cells. circ-PRKCI promoted the viability, migration and invasion of $\mathrm{HCC}$ cells by downregulating the expression levels of miR-1294 and miR-186-5p. In addition, circ-PRKCI was discovered to target miR-1294 and miR-1294 was identified to target FOXK1. Inhibition of circ-PRKCI also 
A

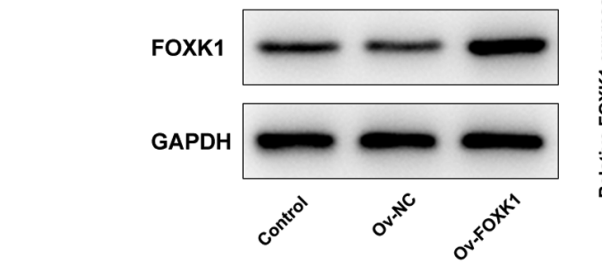

C
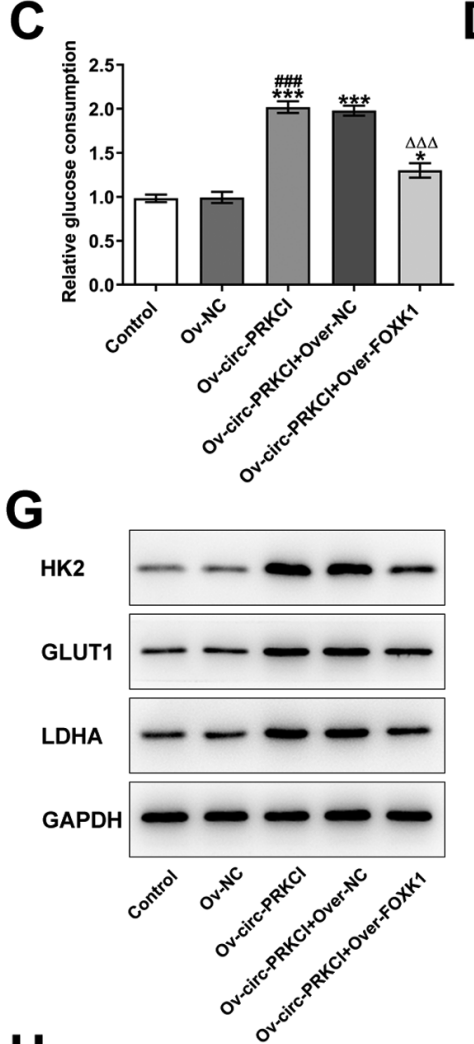

D

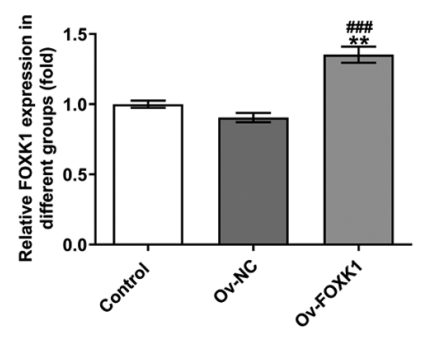

B

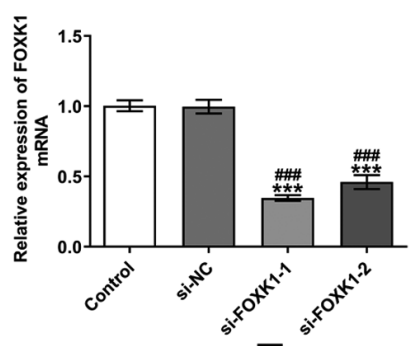

$\mathbf{F}$

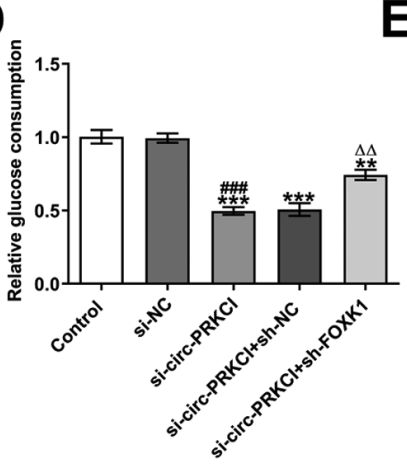

E
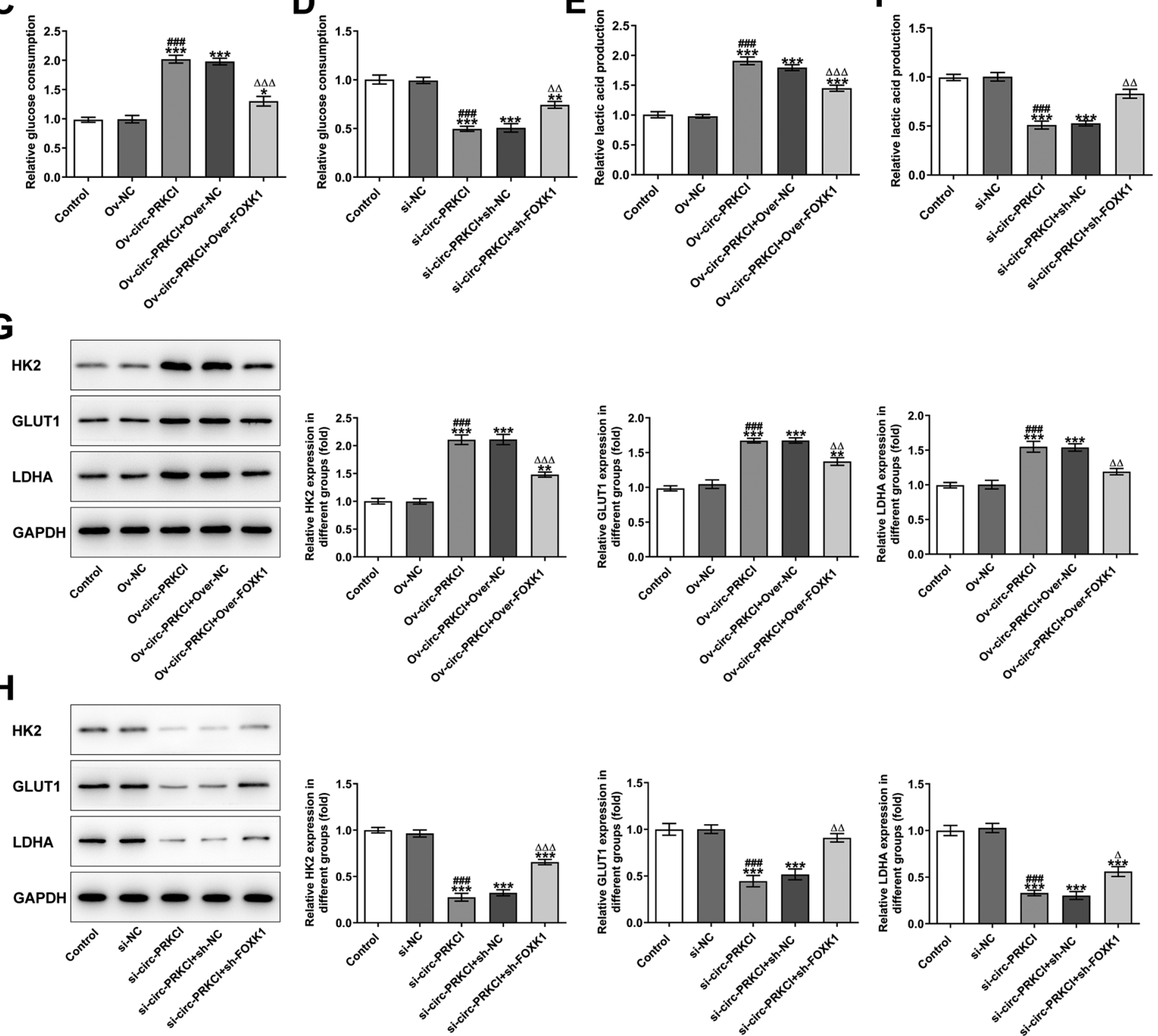

Figure 5. circ-PRKCI suppresses glycolysis in hepatocellular carcinoma cells by targeting miR-1294 and miR-186-5p. (A) FOXK1 expression levels in HCCLM3 cells following the transfection with Ov-FOXK1 were analyzed using western blotting. ${ }^{* *} \mathrm{P}<0.01$ vs. Control; \#\#\# P<0.001 vs. Ov-NC. (B) FOXK1 mRNA expression levels in HCCLM3 cells following the transfection with si-FOXK1-1 and si-FOXK1-2 were analyzed using reverse transcription-quantitative PCR. ${ }^{* * *} \mathrm{P}<0.001$ vs. Control; ${ }^{\# \#} \mathrm{P}<0.001$ vs. si-NC. (C) Glucose levels in the supernatant of HCCLM3 cells following the transfection with (C) Ov-circ-PRKCI and si-FOXK1 and (D) si-circ-PRKCI and Ov-FOXK1 were determined using a commercially available kit. ${ }^{*} \mathrm{P}<0.05,{ }^{* *} \mathrm{P}<0.01,{ }^{* * *} \mathrm{P}<0.001 \mathrm{vs}$. Control; ${ }^{\# \# \#} \mathrm{P}<0.001$ vs. Ov-NC/si-NC; ${ }^{\Delta \Delta} \mathrm{P}<0.01,{ }^{\Delta \Delta \Delta} \mathrm{P}<0.001$ vs. Ov-circ-PRKCI/si-circ-PRKCI. Lactic acid levels in the supernatant of HCCLM3 cells following the transfection with (E) Ov-circ-PRKCI and si-FOXK1 and (F) si-circ-PRKCI and ov-FOXK1 were determined using a commercially available kit. ${ }^{*} *{ }^{2}<0.001$ vs. Control;

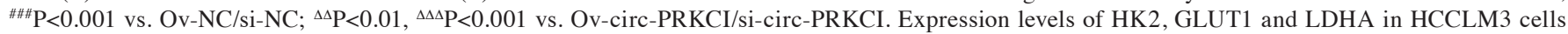
following the transfection with $(\mathrm{G})$ Ov-circ-PRKCI and si-FOXK1 and $(\mathrm{H})$ si-circ-PRKCI and Ov-FOXK1 were analyzed using western blotting. ${ }^{* *} \mathrm{P}<0.01$, ${ }^{* * *} \mathrm{P}<0.001$ vs. Control; ${ }^{\# \#} \mathrm{P}<0.001$ vs. Ov-NC/si-NC; ${ }^{\Delta} \mathrm{P}<0.05,{ }^{\Delta \Delta} \mathrm{P}<0.01,{ }^{\Delta \Delta \Delta} \mathrm{P}<0.001$ vs. Ov-circ-PRKCI/si-circ-PRKCI. Circ, circular RNA; PRKCI, protein kinase C iota; miR, microRNA; FOXK1, forkhead box K1; Ov, overexpression; si, small interfering RNA; NC, negative control; HK2, hexokinase-2; GLUT1, glucose transporter 1; LDHA, lactate dehydrogenase A. 
suppressed glycolysis in the HCC cells by downregulating FOXK1 expression levels via miR-1294. A previous study also showed that the knockdown of FOXK1 suppressed glycolysis in liver cancer cells (15).

In conclusion, the findings of the present study suggested that circ-PRKCI may promote the viability, migration and invasion and glycolysis of HCC cells by upregulating FOXK1 expression levels by targeting miR-1294 and miR-186-5p. These results supported that circ-PRKCI may represent a novel target for the treatment of HCC. Due to the present study being limited by only performing in vitro cell experiments, which do not mimic the in vivo environment, the expression levels of circ-PRKCI, miR-1294 and miR-186-5p will be further investigated in tumor tissues and the present results will be further verified in in vivo animal experiments.

\section{Acknowledgements}

Not applicable.

\section{Funding}

No funding was received.

\section{Availability of data and materials}

The datasets used and/or analyzed during the current study are available from the corresponding author on reasonable request.

\section{Authors' contributions}

GW conceived and designed the study. WC performed the experiments. YL, JZ and WC analyzed and interpreted the data. WC, YL and JZ confirmed the authenticity of all the raw data. All authors read and approved the final manuscript.

\section{Ethics approval and consent to participate}

Not applicable.

\section{Patient consent for participation}

Not applicable.

\section{Competing interests}

The authors declare they have no competing interests.

\section{References}

1. Forner A, Reig M and Bruix J: Hepatocellular carcinoma. Lancet 391: 1301-1314, 2018.

2. Chen W, Zheng R, Baade PD, Zhang S, Zeng H, Bray F, Jemal A, Yu XQ and He J: Cancer statistics in China, 2015. CA Cancer J Clin 66: 115-132, 2016.

3. Gingold JA, Zhu D, Lee DF, Kaseb A and Chen J: Genomic profiling and metabolic homeostasis in primary liver cancers. Trends Mol Med 24: 395-411, 2018.

4. Invenizzi F, Iavarone M, Donato MF, Mazzucco A, Torre M, Conforti S, Rimessi A, Zavaglia C, Schiavon M, Comacchio G, et al: Pulmonary resection for metastasis of hepatocellular carcinoma recurring after liver transplant: An Italian multicenter experience. Front Oncol 10: 381, 2020.
5. Wei CY, Chen PC, Chau GY, Lee RC, Chen PH, Huo TI, Huang YH, Su YH, Hou MC, Wu JC, et al: Comparison of prognosis between surgical resection and transarterial chemoembolization for patients with solitary huge hepatocellular carcinoma. Ann Transl Med 8: 238, 2020.

6. Jeck WR, Sorrentino JA, Wang K, Slevin MK, Burd CE, Liu J, Marzluff WF and Sharpless NE: Circular RNAs are abundant, conserved, and associated with ALU repeats. RNA 19: 141-157, 2013.

7. Wu L, Li Y, Xu XM and Zhu X: Circular RNA circ-PRKCI promotes cell proliferation and invasion by binding to microRNA-545 in gastric cancer. Eur Rev Med Pharmacol Sci 23: 9418-9426, 2019.

8. Shi N, Shan B, Gu B, Song Y, Chu H and Qian L: Circular RNA circ-PRKCI functions as a competitive endogenous RNA to regulate AKT3 expression by sponging miR-3680-3p in esophageal squamous cell carcinoma. J Cell Biochem 120: 10021-10030, 2019.

9. Qi SX, Sun H, Liu H, Yu J, Jiang ZY and Yan P: Role and mechanism of circ-PRKCI in hepatocellular carcinoma. World J Gastroenterol 25: 1964-1974, 2019.

10. Cai X, Yu L, Chen Z, Ye F, Ren Z and Jin P: Arsenic trioxideinduced upregulation of miR-1294 suppresses tumor growth in hepatocellular carcinoma by targeting TEAD1 and PIM1. Cancer Biomark 28: 221-230, 2020.

11. Shan Y and Li P: Long intergenic non-protein coding RNA 665 regulates viability, apoptosis, and autophagy via the MiR-186-5p/ MAP4K3 axis in hepatocellular carcinoma. Yonsei Med J 60: 842-853, 2019.

12. Lan T, Yan X, Li Z, Xu X, Mao Q, Ma W, Hong Z, Chen X and Yuan Y: Long non-coding RNA PVT1 serves as a competing endogenous RNA for miR-186-5p to promote the tumorigenesis and metastasis of hepatocellular carcinoma. Tumour Biol 39: 1010428317705338, 2017.

13. Wang Y, Liu G, Sun S and Qin J: miR-1294 alleviates epithelialmesenchymal transition by repressing FOXK1 in gastric cancer. Genes Genomics 42: 217-224, 2020.

14. Zhang Z, Zhang W, Mao J, Xu Z and Fan M: miR-186-5p functions as a tumor suppressor in human osteosarcoma by targeting FOXK1. Cell Physiol Biochem 52: 553-564, 2019.

15. Cui H, Gao Q, Zhang L, Han F and Wang L: Knockdown of FOXK1 suppresses liver cancer cell viability by inhibiting glycolysis. Life Sci 213: 66-73, 2018.

16. Livak KJ and Schmittgen TD: Analysis of relative gene expression data using real-time quantitative PCR and the 2(-Delta Delta C(T)) Method. Methods 25: 402-408, 2001.

17. Kawauchi K, Araki K, Tobiume K and Tanaka N: p53 regulates glucose metabolism through an IKK-NF-kappaB pathway and inhibits cell transformation. Nat Cell Biol 10: 611-618, 2008.

18. Shi D, Zhao D, Niu P, Zhu Y, Zhou J and Chen H: Glycolysis inhibition via mTOR suppression is a key step in cardamonininduced autophagy in SKOV3 cells. BMC Complement Altern Med 18: 317, 2018.

19. Gillies RJ and Gatenby RA: Hypoxia and adaptive landscapes in the evolution of carcinogenesis. Cancer Metastasis Rev 26: 311-317, 2007.

20. Ding Z, Yang L, Xie X, Xie F, Pan F, Li J, He J and Liang H: Expression and significance of hypoxia-inducible factor-1 alpha and MDR1/P-glycoprotein in human colon carcinoma tissue and cells. J Cancer Res Clin Oncol 136: 1697-1707, 2010.

21. Liberti MV and Locasale JW: The Warburg effect: How does it benefit cancer cells? Trends Biochem Sci 41: 211-218, 2016.

22. Benjamin DI, Cravatt BF and Nomura DK: Global profiling strategies for mapping dysregulated metabolic pathways in cancer. Cell Metab 16: 565-577, 2012.

23. Orang AV, Petersen J, McKinnon RA and Michael MZ: Micromanaging aerobic respiration and glycolysis in cancer cells. Mol Metab 23: 98-126, 2019.

24. Guo W, Qiu Z, Wang Z, Wang Q, Tan N, Chen T, Chen Z, Huang S, Gu J, Li J, et al: MiR-199a-5p is negatively associated with malignancies and regulates glycolysis and lactate production by targeting hexokinase 2 in liver cancer. Hepatology 62: 1132-1144, 2015.

25. Iansante V, Choy PM, Fung SW, Liu Y, Chai JG, Dyson J, Del Rio A, D'Santos C, Williams R, Chokshi S, et al: PARP14 promotes the Warburg effect in hepatocellular carcinoma by inhibiting JNK1-dependent PKM2 phosphorylation and activation. Nat Commun 6: 7882, 2015. 
26. Liang Y, Zhang D, Zheng T, Yang G, Wang J, Meng F, Liu Y, Zhang G, Zhang L, Han J, et al: IncRNA-SOX2OT promotes hepatocellular carcinoma invasion and metastasis through miR122-5p-mediated activation of PKM2. Oncogenesis 9: 54, 2020.

27. Xiao Q, Wei Z, Li Y, Zhou X, Chen J, Wang T, Shao G, Zhang M and Zhang Z: miR-186 functions as a tumor suppressor in osteosarcoma cells by suppressing the malignant phenotype and aerobic glycolysis. Oncol Rep 39: 2703-2710, 2018.

28. Liu Y, Jiang Y, Xu L, Qu C, Zhang L, Xiao X, Chen W, Li K, Liang Q and Wu H: circ-NRIP1 promotes glycolysis and tumor progression by regulating miR-186-5p/MYH9 axis in gastric cancer. Cancer Manag Res 12: 5945-5956, 2020.

29. Sukonina V, Ma H, Zhang W, Bartesaghi S, Subhash S, Heglind M, Foyn H, Betz MJ, Nilsson D, Lidell ME, et al: FOXK1 and FOXK2 regulate aerobic glycolysis. Nature 566: 279-283, 2019
30. Zhang Z, Lin W, Gao L, Chen K, Yang C, Zhuang L, Peng S, Kang M and Lin J: Hsa_circ_0004370 promotes esophageal cancer progression through miR-1294/LASP1 pathway. Biosci Rep 39: BSR20182377, 2019.

31. Guo TY, Xu HY, Chen WJ, Wu MX and Dai X: Downregulation of miR-1294 associates with prognosis and tumor progression in epithelial ovarian cancer. Eur Rev Med Pharmacol Sci 22: 7646-7652, 2018

32. Kan XQ, Li YB, He B, Cheng S, Wei Y and Sun J: MiR-1294 acts as a tumor inhibitor in cervical cancer by regulating FLOT1 expression. J Biol Regul Homeost Agents: Apr 24, 2020 (Epub ahead of print).

(i) (3) $(-)$ This work is licensed under a Creative Commons Attribution-NonCommercial-NoDerivatives 4.0 International (CC BY-NC-ND 4.0) License. 